\title{
VECTOR CONTROL OF INDUCTION MOTOR WITH SPLIT PHASE
}

\section{STATOR WINDINGS}

\section{K. Gopakumar}

CEDT

Research student

\section{V.T.Ranganathan}

E.E

Professor
S.R.Bhat

CEDT

Professor

\section{INDIAN INSTITUTE OF SCIENCE \\ BANGALORE - 560012}

INDIA

\section{ABSTRACT}

A vector controlled scheme is described for induction motor with split phase windings. Such a motor is obtained by splitting the phase windings of a conventional three phase motor with an angular seperation of 30 electrical degrees between the axes of the two halves. In the proposed scheme the motor is run as a three phase machine by connnecting the split phase windings in series. However the winding taps are utilised for making voltage measurements. Based on a space phasor model of the split phase machine, a method of acquiring the position of the rotor flux is developed. The scheme is based only on the voltage and current measurements, without the need for position encoders. It is also independent of machine resistance values. Results from computer simulation as well as from an experimental prototype drive are presented and discussed.

\section{Introduction}

Split phase induction motors are used in conjunction with current source inverters, inorder to reduce the low frequency torque ripple and also to extend the high frequency operation of a CSI fed induction motor drive [1][2]. A split phase motor configuration can be easily obtained by splitting the phase belts of a conventional 3-phase motor into two equal halves with an angular separtion of 30 electrical degrees [3]. In current source inverter drives two independent inverters are used to drive the split phase group of coils [1][2][3]. In the present study the split phase motor configuration is studied using voltage source inverters, by running the motor as a 3-phase configuration by appropriately connecting the split phase stator groups Fig l.b [3]. Here the split phase stator groups are used for voltage measurements for a rotor flux estimation, from terminal measurements of voltages and currents only. In the following section the motor dynamic model equations are presented in space phasor notations

\section{Split phase induction motor model}

The schematic representation of the split phase motor is shown in Fig la and Fig lb shows the schematic representation for the three phase operation of the split phase motor. The slot wise distribution of conductors of the different phase groups of the split phase motor used for the present study is shown in Fig 2. Conductors of different phase groups come in the same slot due to the split phase stator structure, resulting in a leakage flux coupling between conductors of different phase groups [4][6]. Taking this leakage flux coupling into consideration, the voltage space phasor based equation for the motor can be written as

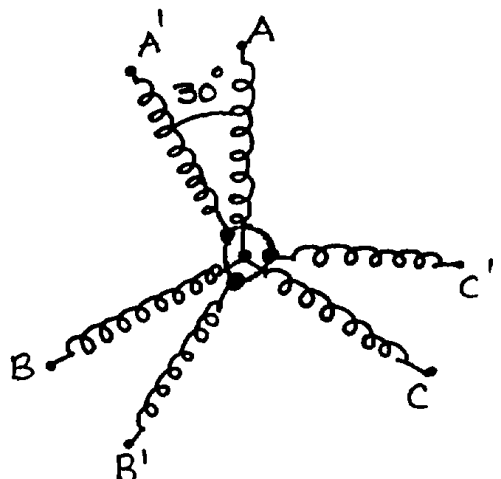

Fig - 1a. Split phase induction motor stator - schematic representation.

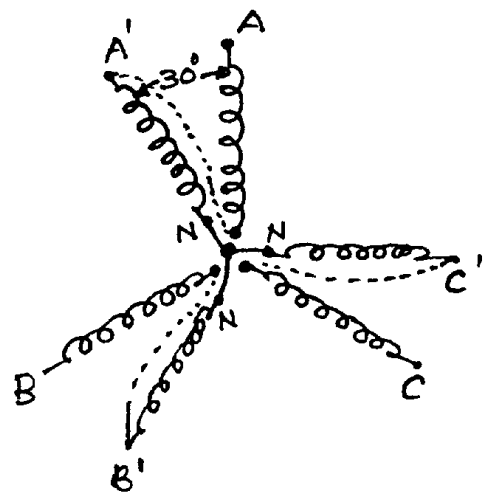

Fig - $1 \mathrm{~b}$. Interconnection of split phase motor stator for 3-phase configuration.

A'B'C' Phase Group

$$
\begin{aligned}
& R_{S} \underline{i_{S 1}}(t)+L_{S S} \frac{d i_{S 1}(t)}{d t}+\left(1-\sigma_{12}\right) L_{S S} \frac{d i_{S S}(t)}{d t} e^{-j 30^{0}}+ \\
& L_{S R} \frac{d i_{Y}^{S}(t)}{d t} e^{-j 15^{0}}=\underline{v_{S 1}}(t)-(1)
\end{aligned}
$$

ABC Phase Group

$$
\begin{aligned}
R_{S} \underline{i_{S 2}}(t)+L_{S S} \frac{d i_{S Z(t)}}{d t}+\left(1-\sigma_{12}\right) L_{S S} \frac{d i_{S S(1)}}{d t} e^{j 30^{\circ}}+ \\
L_{S R} \frac{d j_{S}^{S}(t)}{d t} e^{j 15^{0}}=\underline{v_{S 2}(t)-(2)}
\end{aligned}
$$




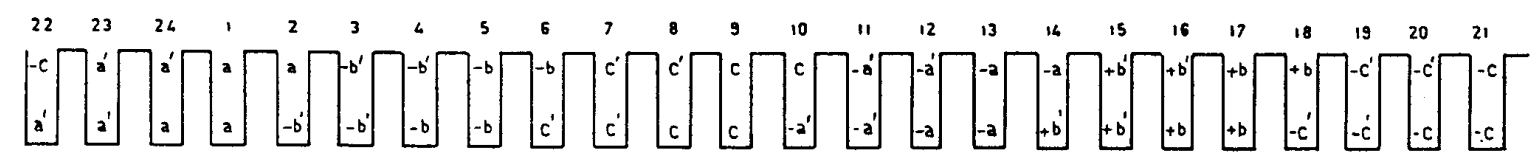

Fig - 2. Slot wise distribution of conductors - split phase induction motor.

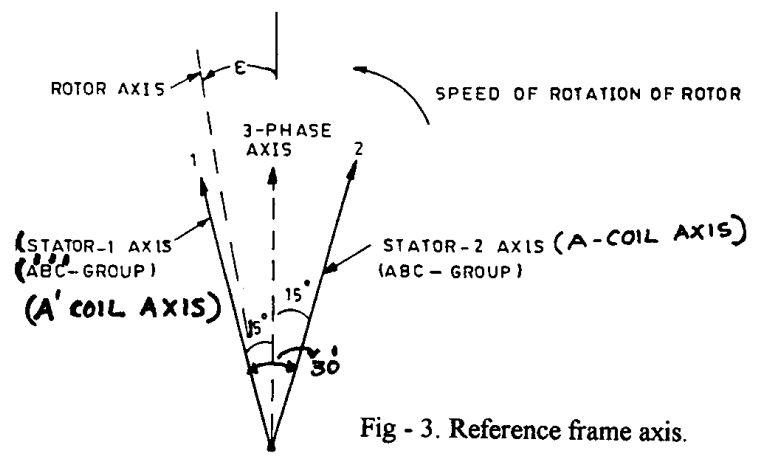

$\underline{\text { Rotor }}$

$$
\begin{aligned}
& R_{r} \underline{i_{r}}(t) e^{-j \varepsilon}+L_{r r} \frac{d}{d t} i_{\underline{r}}^{S}(t) e^{-j \varepsilon}+L_{S R} \frac{d}{d t} i_{S \underline{S 1}}(t) e^{j(15-\varepsilon)}+ \\
& \underline{i}_{S 2}(t) e-j(15+\varepsilon)=0-(3)
\end{aligned}
$$

In equation (1), the voltage space phasor equation for the $A^{\prime} B^{\prime} C^{\prime}$ phase group is presented, in which the reference axis (real component $-\alpha$ ) for the $A^{\prime} B^{\prime} C^{\prime}$ phase group is placed along the $A^{\prime}$ - phase coil axis and the imaginary axis $(\beta)$ is placed perpendicular to the $A^{\prime}$ - phase coil axis (Fig 3). Correspondingly the reference axis for the $\mathrm{ABC}$ phase group is placed along A - phase coil axis. In equation (3) the voltage space phasor equation for the rotor circuit is defined with respect to a reference axis ( 3 phase reference axis) placed mid way between the $\mathrm{A}$ - phase and $\mathrm{A}^{\prime}$ - phase reference axis (Fig 3 ).

where,

$\underline{i_{S 1}}(t), \underline{v_{S 1}}(t)$ - current and voltage space phasors associated with A'B'C' phase group

$\underline{i_{S 2}}(t), v_{S 2}(t)-$ cuurent and voltage space phasors associate with $\mathrm{ABC}$ phase grou

$\epsilon-$ rotor position with respect 3 - pahse reference axis

$R_{S}-$ split phase stator resistance per phase

$L_{S S}$ - split phase stator inductance per phas

$L_{S R}$ - split phase (stator referred) stator to rotor leakage inductance per phase

$L r r$ - split phase stator referred rotor self inductance per phase.

$\left(1-\sigma_{12}\right) L_{S S}-$ split phase stator mutual inductance coupling

coefficient term between split phase stator groups,

including the leakage coupling coeffficient

$R_{r}$ - stator referred rotor resistance per phase

$i_{r}^{S}(t)$ - stator referred rotor current space phasor

In equation (1), equation (2) and equation (3) the space phasor based equations are written for current and voltage of any general variation with respect to time and can be re-written for sinusoidal excitation [5].

\section{Sinusoidal steady state equivalent circuit model}

The motor equations (eqn (1), eqn (2), eqn (3) ) under sinusoidal steady state excitation are [5]

\section{$\underline{A^{\prime} B C^{\prime}}$ phase group}

$$
\begin{gathered}
R_{S} \underline{I}_{A}^{\prime}+j \omega L_{S S} I_{A}^{\prime}+j \omega\left(1-\sigma_{12}\right) L_{S S} \underline{A}_{A} e^{-j 30^{0}} \\
+j \omega L_{S R} I_{\underline{R}} e^{-j 15^{0}}=V_{A}^{\prime}
\end{gathered}
$$

\section{$\underline{A B C}$ phase group}

$$
\begin{aligned}
R_{S} \underline{I}_{A}+j \omega L_{S S} I_{A} & +j \omega\left(1-\sigma_{12}\right) L_{S S} I_{A}^{\prime} e^{j 30^{0}} \\
& +j \omega L_{S R} I_{R} e^{j 15^{0}}=V_{A}-(5)
\end{aligned}
$$

$\underline{\text { Rotor }}$

$$
\begin{array}{r}
R_{r} \underline{I}_{R}+j \omega_{S l} L_{r r} \underline{I}_{R}+j \omega_{S l}\left(\underline{I}_{A}^{\prime} e^{j 15^{0}}+\underline{I_{A}} e^{-j 15^{0}}\right) \\
=0
\end{array}
$$

where

$I_{A}^{\prime}, V_{A}^{\prime}$ - stator current and voltage space phasor under sinusoidal excitation for the $A^{\prime} B^{\prime} C^{\prime}$ ' phase group

$I_{A}, V_{A}-$ - current and voltage space phasor under sinusoidal excitation for the $\mathrm{ABC}$ phase group

$I_{R} \longrightarrow$ rotor current space phasor under sinusoidal excitation

$\bar{\omega}_{s l}-$ slip frequency

$\omega$ - synchronous frequency

The rortor equation ( eqn - 6 ) can be written as

$\frac{R_{r}}{S} \underline{I_{R}}+j \omega L_{r r} \underline{I_{R}}+j \omega L_{S R}\left(I_{A}^{\prime} e^{e 15^{0}}+\underline{I}_{A} e^{-j 15^{0}}\right)=0$

where

$S=\frac{\omega_{s l}}{\omega}$

Equations (4), (5) and (7) reprent the split phase motor model under sinusoidal excitation and can be defined with respect to a common reference axis (Fig.3) (3-phase reference axis ) by multiplying eqn (4) by $e^{j 15^{0}}$ and eqn (5) by $e^{-j 15^{0}}$ i.e, the voltage space phasor equation for the $\mathrm{A}^{\prime} \mathrm{B}^{\prime} \mathrm{C}^{\prime}$ phase group defined with respect to the 3-phase reference axis ( Fig.3 )

$$
\begin{gathered}
R_{S} I_{A}^{\prime} e^{j 15^{0}}+j \omega L_{S S} I_{A}^{\prime} e^{j 15^{0}}+j \omega\left(1-\sigma_{12}\right) L_{S S} I_{A} e^{-j 15^{0}} \\
+j \omega L_{S R} I_{R}=V_{A}^{\prime} e^{j 15^{0}}
\end{gathered}
$$

Voltage space phasor equation for the $\mathrm{ABC}$ phase group defined with respect to the 3-phase reference axis in ( Fig.3)

$$
\begin{gathered}
R_{S} \underline{I}_{A} e^{-j 15^{0}}+j \omega L_{S S} \underline{I}_{A} e^{-j 15^{0}}+j \omega\left(1-\sigma_{12}\right) L_{S S} \underline{I}_{A} e^{j 15^{0}} \\
+j \omega L_{S R} \underline{I_{R}}=V_{A} e^{-j 15^{0}}
\end{gathered}
$$

From eqn (7), eqn (8) and eqn (9) a steady state motor model 570 for the split phase motor can be prposed and is shown in Fig(4) -4 
[4]. The split phase motor model proposed is also valid for the three phase operation (Fig 1.b) wherein the current space phasors $i_{S 1}(t)$ will be equal to $i_{S 2}(t)$. This implies that the real component of $i_{S 1}(t)$ is equal to the real component of $i_{S 2}(t)$ is equal to the imaginary component of $i_{S 2}(t)$ under three phase operation. The split phase motor model parameters can be found out by running the motor as a 3-phase motor ( Fig 1.b) and conducting the open circuit and short circuit test. The stator rsistance $R_{S}$ can be found out from DC measurement and the $R_{r}$ can be found out from measuring the input power under short circuit operation.

\section{Open circuit test}

The motor is run as a 3-phase motor and the split phase motor voltages are measured with respect to the common current

\section{$\left(\mathrm{A}^{\prime} \mathrm{B}^{\prime} \mathrm{C}^{\prime}\right)$ phase group}

$$
\begin{aligned}
R_{S} I_{A} e^{j 15^{0}}+j \omega L_{S S} I_{A} e^{j 15^{0}}+j \omega\left(1-\sigma_{12}\right) L_{S S} I_{A} e^{-j 15^{0}} & \\
= & V_{A}^{\prime} e^{j 15^{0}}-
\end{aligned}
$$

where

$I_{A}^{\prime}=I_{A}$ (3-phase operation)

\section{$(\mathrm{ABC})$ pahse group}

$$
\begin{array}{r}
R_{S} I_{A} e^{-j 15^{0}}+j \omega L_{S S} \underline{I}_{A} e^{-j 15^{0}}+j \omega\left(1-\sigma_{12}\right) L_{S S} \underline{I}_{A} e^{j 15^{0}} \\
=\underline{V}_{A} e^{-j 15^{0}}-(11
\end{array}
$$

Knowing the value of $R_{S}$ from DC measurement, subtracting eqn(11) multiplied by $e^{j 15^{0}}$ from eqn (10) multiplied by $e^{-j 15^{\circ}}$ gives the value of $\left(1-\sigma_{12}\right) L_{S S}$. Once $R_{S}$ and $\left(1-\sigma_{12}\right) L_{S S}$ is known $L_{S S}$ can be found out from eqn (10) and eqn (11).

\section{Short circuit test}

The $\left(A^{\prime} B^{\prime} C^{\prime}\right)$ pahse group voltage equation under short circuit can be written from Fig(4). ie.

$$
\begin{aligned}
& R_{S} \underline{I}_{A} e^{j 15^{0}}+j \omega \sigma_{12} L_{S S} \underline{I}_{A} e^{j 15^{0}}+I_{A}\left(e^{j 15^{0}}+e^{-j 15^{0}}\right) \times \\
& {\left[\left(1-\sigma_{12}\right) j \omega L_{S S}-j \omega L_{S R}+j \omega L_{l r}+R_{r}\right]=V_{A}^{\prime} e^{j 15^{0}}-}
\end{aligned}
$$

(ABC) phase group

$$
\begin{aligned}
& R_{S} \underline{I}_{A} e^{-j 15^{0}}+j \omega \sigma_{12} L_{S S} I_{A} e^{-j 15^{0}}+I_{A}\left(e^{j 15^{0}}+e^{-j 15^{0}}\right) \times \\
& {\left[\left(1-\sigma_{12}\right) j \omega L_{S S}-j \omega L_{S R}+j \omega L_{l r}+R_{r}\right]=V_{A} e^{-j 15^{0}}}
\end{aligned}
$$

From eqn (12) and eqn (13) from the previously calculated vales of $R_{S}, R_{r},\left(1-\sigma_{12}\right) L_{S S}$ and $L_{S S}$, the term $\left(\left(1-\sigma_{12}\right) L_{S S}-L_{S R}\right)+L_{l}$ can be found out from short circuit test and is equally divided between the rotor leakage component $L_{i r}$ and the stator leakage coupling inductance $\left(\left(1-\sigma_{12}\right) L_{S S}-L_{S R}\right)$. The motor model parameters obtained from the IHP split phase induction motor used for the present experimental setup are shown in Appendix A. From the motor model parameters and using the split phase voltage space phasor equations ( eqn (1) and eqn (2)), a rotor flux sensing scheme is presented in the following sections

\section{Rotor Flux Position Estimation}

From Fig (4) and from equation (1) and equation (2) the rotor flux space phasor $\left(\Psi_{r}(t)\right)$ in stationary co-ordinates, defined with respect to the 3 phase reference (Fig 3) axis can be written as ,

$\underline{\Psi}_{r}(t)=L_{r r} \underline{i}_{r}^{s}(t)+L_{S R}\left(i_{S 1}(t) e^{j 15^{\circ}}+\underline{i}_{S 2}(t) e^{-j 15^{o}}\right)$

Now from equation (14),

$\underline{i_{r}^{s}}(t)=\frac{1}{L_{r r}}\left(\Psi_{r}(t)-L_{S R}\left(i_{S 1}(t) e^{j 15^{\circ}}+i_{S 2}(t) e^{-j 15^{\circ}}\right)\right)$

When the motor is run as a three phase motor, the current space phasors are identical and is equal to, $(F, g .1 \mathrm{~b})$

$\underline{i s 1}_{\underline{1}}(t)=i_{s 2}(t)=i_{s}$

Substituting eqn.(14) and eqn.(15) in eqn (1) and eqn.(2) and re-arranging in real and imaginary components gives,

$L_{s S}\left(\sigma-\sigma_{12}\right) \frac{d}{d t} i_{s \beta}(t)-2 \sin 15^{\circ} \frac{L_{S R}}{L r} \frac{d}{d t} \Psi_{\gamma \beta}(t)=v_{S 2 \alpha}(t)-v_{S 1 \alpha}(t)$

and

$L_{S S}\left(\sigma-\sigma_{12}\right) \frac{d}{d t} i_{S \alpha}(t)+2 \sin 15^{0} \frac{L_{S R}}{L_{r}} \frac{d}{d t} \Psi_{r \alpha}(t)=v_{S 2 \beta}(t)-v_{S 1 \beta}(t)$

From eqn.(16) and eqn.(17)

$\Psi_{r \beta}(t)=\frac{L_{r}}{L_{S R} 2 \sin 15^{\circ}}\left[\int\left(v_{S 1 \alpha}(t)-v_{S 2 \alpha}(t)\right)-\left(\sigma-\sigma_{12}\right) L_{S S} i_{S \beta}(t)\right]-$
$\ldots$ (18)

$\Psi_{r \alpha}(t)=\frac{L_{r}}{L_{S R} 2 \sin 15^{\circ}}\left[\int\left(v_{S 2 \beta}(t)-v_{S] \beta}(t)\right)-\left(\sigma-\sigma_{12}\right) L_{S S} i_{S \alpha}(t)\right]-$

where, $\quad \sigma=\left(1-\frac{L_{S R}^{2}}{L_{S S} L_{r}}\right), i_{S}(t)=i_{S \alpha}(t)+j i_{S \beta}(t)$,

$\underline{\Psi}_{r}(t)=\Psi_{r \alpha}(t)+j \Psi_{\text {r阝 }}(t), \quad \underline{v}_{S 2}(t)=v_{S 2 \alpha}(t)+j v_{S 2 \beta}(t)$

$\underline{v_{S 1}}(t)=v_{S 1 \alpha}(t)+j v_{S 1 \beta}(t)$

From eqn.(18.a) and eqn.(18.b) $\Psi_{r \alpha}(t)$ and $\psi_{r_{\beta}}(t)$ can be computed from measuring the split -phase voltages and currents and from $\psi_{r \alpha}(t)$ and $\psi_{r \beta}(t)$ the rotor flux position can be easily estimated.

$$
\begin{aligned}
& \text { i.e., } \\
& \cos \rho=\frac{\Psi_{\text {ra }}(t)}{\sqrt{\left(\Psi_{\text {ra }}(t)\right)^{2}+\left(\Psi_{r \beta}(t)\right)^{2}}}--(19 . a) \\
& \sin \rho=\frac{\Psi_{\text {r } \beta}(t)}{\sqrt{\left(\Psi_{\text {ra }}(t)\right)^{2}+\left(\Psi_{r \beta}(t)\right)^{2}}}--(19 . b)
\end{aligned}
$$




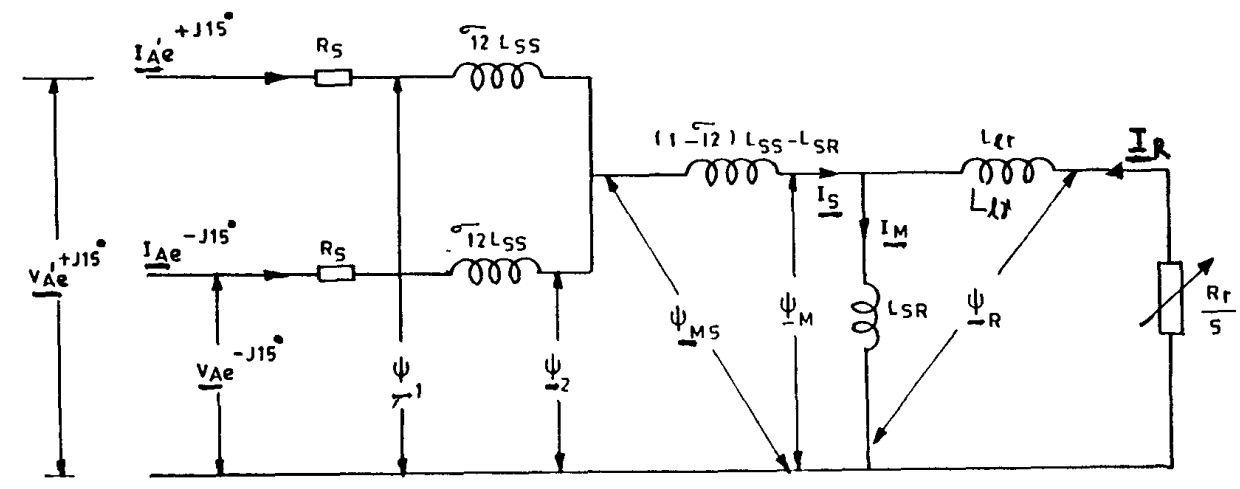

Fig - 4. Steady state equivalent circuit.

\section{Simulation and Experimental Result}

A field oriented control scheme for the split phase motor configuration is implemented for $1 \mathrm{HP}$ motor developed for the present study Fig(5). The proposed scheme is simulated using simulation package TUTSIM . Fig 6 shows the simulation results of the rotor flux, speed and torque producing current component for a speed reversal and Fig.7 shows the signal variation for a step change in load torque. The simulated results are also compared and verified using an experimental set up. Fig.(8.a) shows the speed signal and the rotor flux signal for a speed reversal and Fig.(8.b) shows the speed and flux signal during speeding up. Fig. (9) shows the flux signal and the torque producing current component for a step change of load torque. Fig.(10.a) shows the speed signal and the torque current component for a step change of load torque. Fig 10.b shows phase-A current and torque component current during a step change in load. Fig.(11) shows the phase - A and phase - B currents. The simulation results as well as the experimental results confirm that decoupled control of the split phase induction motor is possible from terminal measurements of voltages and currents without any requirement for any special encoders.

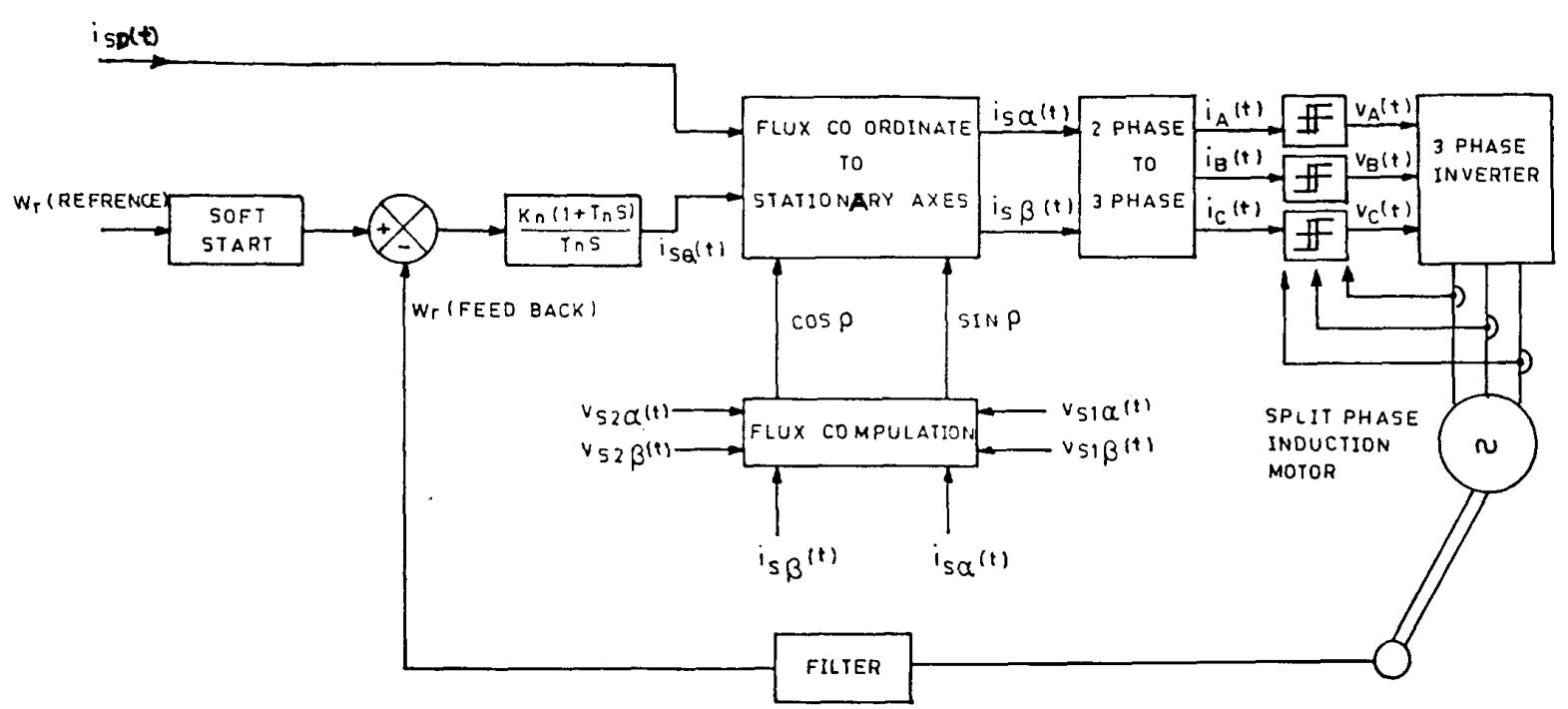

Fig - 5. - Field oriented control scheme-split phase motor drive 


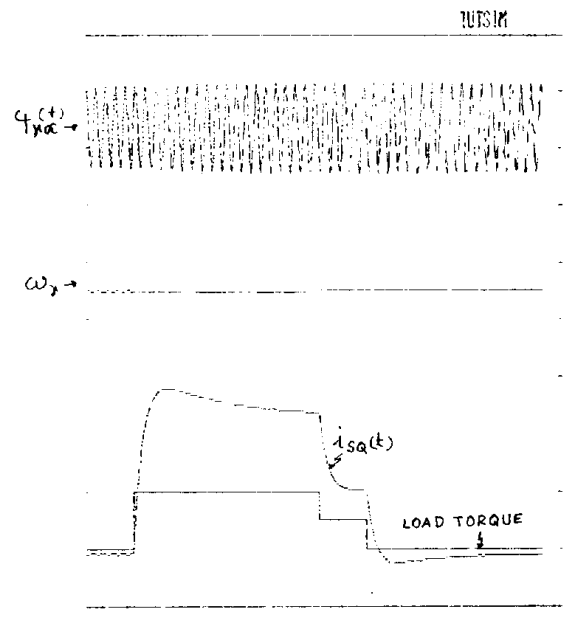

Fig - 7. Rotor flux, speed and torque component current under variable load operation simulated result.

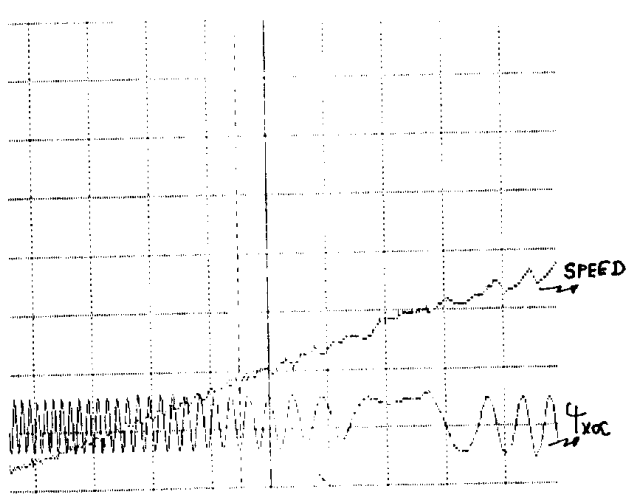

Fig - 8a. Speed signal and rotor flux signal under speed reversal - Experimental result.

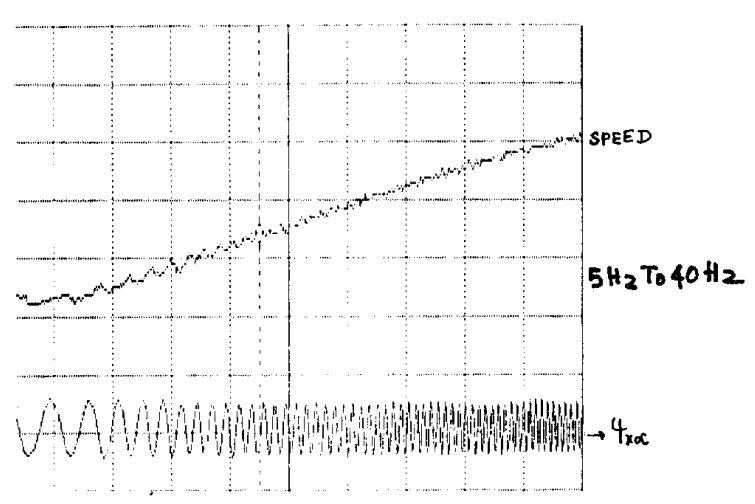

Fig - 8b. Speed and flux during acceleration - Experimental result.

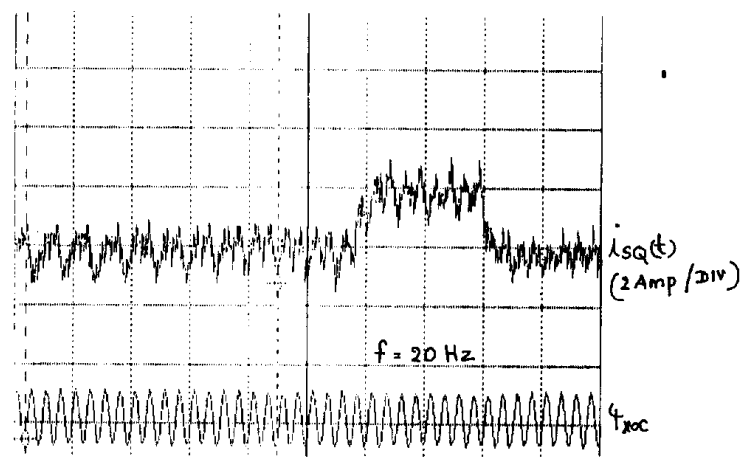

Fig - 9. Flux and torque component current during step change in load - Experimental result.

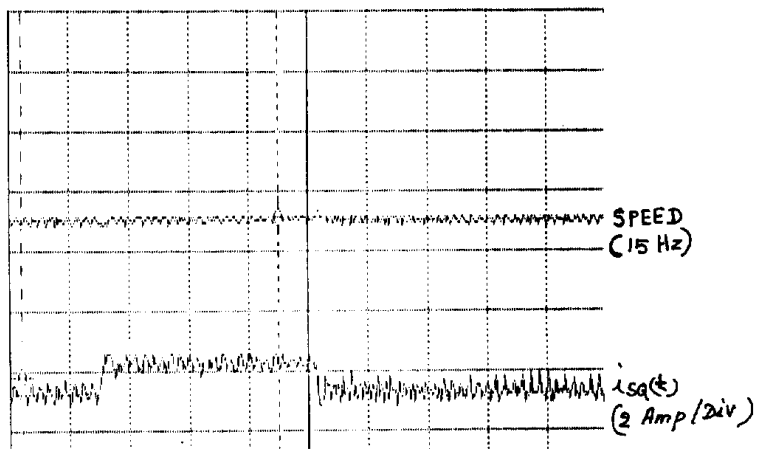

Fig - 10a. Speed and torque component current during step change in load - Experimental result.

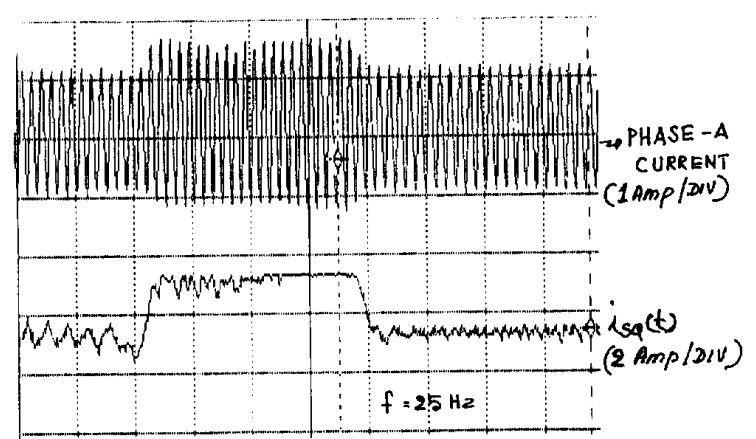

Fig - 10b. Phase - A current and torque component current during step change in load - Experimental result.

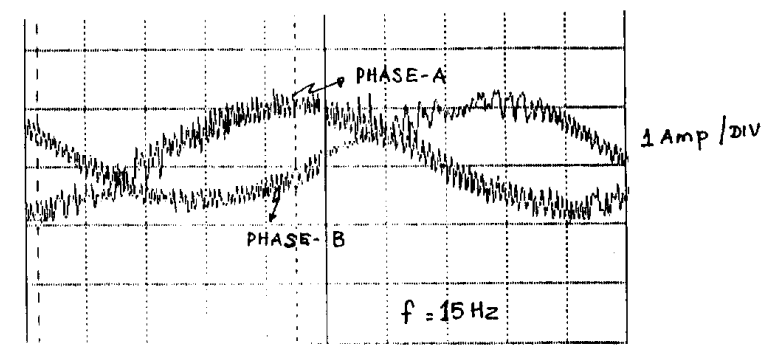

Fig - 11. Phase - A and phase - $A^{\prime}$ current wave form during 573 steady state operation - Experimental result. 


\section{Conclusion}

A field oriented control scheme, using split - phase voltage and current measurements, for an induction motor drive is presented. An equivalent circuit model based on space phasor definitions is presented. The proposed scheme is analysed using computer simulation and also verified using experimental results. The salient features of the proposed scheme are,

- Rotor flux position estimation is possible from split - phase terminal voltage and current measurements for an induction motor

- Costly and complicated position encoders are not required.

- Motor resistance parameters are not involved in the flux computation.

- Voltage sensing from the conventional induction motor phase belt tapping is required and splitting the phase can be easily achieved from conventional motors with even number of slots per pole.

\section{Reference}

1. T.A.Lipo : Design and control techniques for extending high frequency operation of a CSI induction motor drive, IEEE - IAS, conf, pp. 698-705, 1982

2. E.Andersion, K.Bieniek: Six-phase motors for current source inverter drives, IEEE, IAS, conf, pp 607-612, 1981.

3. K.Gopakumar, S.Sathiakumar, S.K.Biswas, Joseph Vithayathil: Modified current source inverter fed induction motor drive with reduced torque pulsation, IEEE proceedings, Vol.131, pt.B, No.4, July 1984.

4. T.A.Lipo : A d - q model for six phase induction machines, in proc. Int. conf. Electrical machines, Athens, Greece, 1980.

5. W.Leonhard : "Control of Electrical Drives" Springer Verlag - 1985.

6. Lurong Ye, Longya $\mathrm{Xu}$ : Analysis of a novel stator winding structure minimizing harmonic current and torque ripple for dual siz-step converter-fed high power AC machines, IEEE-IAS, conf-1993. pp. 197-202

\section{Appendix - A}

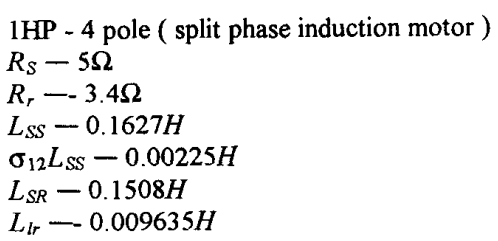

